\title{
Water-based Tai Chi: theoretical benefits in musculoskeletal diseases. Current evidence
}

\author{
Salvador Israel Macías-Hernández',* , Lucio Vázquez-Torres², Juan Daniel Morones-Alba ${ }^{3}$, Roberto Coronado-Zarco ${ }^{4}$, \\ María de los Angeles Soria-Bastida', Eva Cruz-Medina ${ }^{4}$, Tania Inés Nava-Bringas ${ }^{4}$ \\ 'Department of Osteoarticular Rehabilitation, National Institute of Rehabilitation, México City, México \\ ${ }^{2}$ National Model for Investigation, Rehabilitation, and Educational Integration Center "Gaby Brimmer", México City, México \\ ${ }^{3}$ Postgraduate Division. National Institute of Rehabilitation, México City, México \\ ${ }^{4}$ Department of Orthopedic Rehabilitation, National Institute of Rehabilitation, México City, México
}

Tai Chi is a low-impact and moderate intensity exercise that has shown positive effects in patients with musculoskeletal disorders. Recently have been developed clinical studies on the benefits of Tai Chi techniques combined with hydrotherapy. Both types of treatment include physical training of balance, mobility, strength, coordination and sensory input that could complement each other. This report aims to present the current evidence about the benefits of the combination of water based Tai Chi in musculoskeletal diseases in order to establish whether the combined intervention is better than Tai Chi or hydrotherapy alone.

Keywords: Tai Chi, Hydrotherapy, Rehabilitation, Therapeutics, Musculoskeletal diseases

\section{INTRODUCTION}

Tai Chi (TC), also known as Tai Chi Chuan, is a traditional Chinese martial art originally created for self-defense, although today it is considered a low-impact aerobic exercise (Zhang et al., 2012).

Its practice consists of fluid, circular, smooth, and slow movements, with the practitioner in a semi-flexed position of the knees. The practice of this exercise requires precise and slow movements of the joints, the maintenance of postural stability, and balance (Chen et al., 2008), but also meditative aspects that are combined with breathing exercises (Wang et al., 2014).

There are five main styles of TC: Yang; Wu; Chen; Hao, and Sun. Each has its different focus with regard to the movements, but all share the same essential principles of the practice (Lan et al., 2013).

In investigation studies, the 108-movement Yang-style TC has been utilized, as well as simpler and shorter forms of TC of between 8 and 108 movements or other Western variants (Lan et al., 2013).

\section{PHYSIOLOGICAL RESPONSES}

Various published studies have calculated that the expenditure of energy during the practice of TC is between 4.1 and 6.7. Metabolic equivalents (MET) according to the style of the practice (Schneider and Leung, 1991; Zhuo et al., 1984). Lan and collaborators quantified Cardiac frequency (CF) and Oxygen consumption $\left(\mathrm{VO}_{2}\right)$ with the practice of TC in adults; mean $\mathrm{CF}$ was $58 \%$ of the Cardiac frequency reserve (CFR) and a maximal $\mathrm{VO}_{2} 55 \%$. Thus, according to these parameters, TC can be considered an aerobic exercise of moderate intensity (CFR, 40-59\%) (Lan et al., 2001).

\section{THERAPEUTIC USEFULNESS}

There is recent evidence that supports the therapeutic effects of TC for musculoskeletal, rheumatological, neurological, and geriatric conditions (Wang, 2011; 2012). Significant improvements have been reported in balance, muscular strength, cardiorespirato-
*Corresponding author: Salvador Israel Macías-Hernández

Department of Osteoarticular Rehabilitation. Instituto Nacional de Rehabilitación. Av. México-Xochimilco 289, Col. Arenal de Guadalupe, Delegación Tlalpan, 14389

México, D.F.

Tel: +1-52-55-5999-1000, Fax: +1-52-55-5999-1000, E-mail: drisraelmacias@gmail.com Received: May 29, 2015 / Accepted: June 8, 2015
This is an Open Access article distributed under the terms of the Creative Commons Attribution Non-Commercial License (http://creativecommons.org/licenses/by-nc/3.0/) which permits unrestricted non-commercial use, distribution, and reproduction in any medium, provided the original work is properly cited. 
ry function, joint and muscle flexibility, pain, and even symptoms of depression and anxiety (Wang, 2012). In a study that reported the neuromuscular effects of TC in elderly subjects with alterations in equilibrium, significant progresses were demonstrated in neuromuscular control of the knee and in the organization of muscular response during walking, as well as significant improvement with respect to functional equilibrium appraised with different scales (Gatts et al., 2007). There is evidence that TC reduced the incidence of falls in older adults. Possible mechanisms include improvement in equilibrium, caregiving, postural control, propioceptive sensitivity, and reduction of the fear of falling (Hall et al., 2009; Lan et al., 2013). Subjects trained in TC additionally present improved vestibular reactions in sensorial tests during postural changes and greater propioceptive accuracy when compared with the controls (Fong and Ng, 2006; Gyllensten et al., 2010).

A systematic review and meta-analysis published in 2011 on the effects of TC in Osteoarthritis (OA) included six Randomized Clinical Trials (RCT) that compared TC with controls and that concluded that TC was superior with respect to pain, functionality, and joint rigidity on comparing the former with conventional exercise programs or those carried out at home (Kang et al., 2011). Another systematic review, this one published in 2014, on the effects of TC on OA of the knee included six good-quality RCT. The authors concluded that TC exhibited effectiveness in diminution of pain and improvement in physical function, although the review emphasized the need for conducting RCT with larger sample sizes and longer follow-ups to confirm the efficacy of this intervention (Ye et al., 2014).

In a recently published systematic review, the effectiveness was evaluated of physical therapy on improvement of equilibrium and reduction in the risk of falls in patients with $\mathrm{OA}$ of the knee. Fifteen RCT and 1,482 patients were included in the meta-analysis. The interventions taken into account included TC, strength training, aerobic exercise, and aquatic exercise. The study concluded that TC, strength training exercises, and aerobic training improved balance and diminished the risk of falls in older adults with OA of the knee (Mat et al., 2015).

In the guidelines from the European League Against Rheumatism (EULAR, 2013) for non-pharmacological management knee and hip OA, it is included the recommendation to practice TC in patients with $\mathrm{OA}$, based on a systematic review of 10 good quality RCT, where evidence was found of the usefulness of TC in pain reduction, with an effect size of 0.28 up to 1.67 (Escalante et al., 2010; Fernandes et al., 2013).

In 2012, the American College of Rheumatology (ACR) estab- lished a conditioned recommendation on the practice of TC as non-pharmacological treatment of $\mathrm{OA}$ of the knee (Hochberg et al., 2012). Another international association, the Osteoarthritis Research Society International (OARSI), in its guidelines of conservative management of $\mathrm{OA}$, does not establish recommendations with respect to the prescription or use of TC (McAlindon et al., 2014). Recommendations have not been established for these agencies on the usefulness of TC for other pathologies.

With regard to Quality of life (QOL), there is a systematic review on the effectiveness of TC in chronic pathologies that includes $21 \mathrm{RCT}$ and 1,200 patients in a meta-analysis showing positive effects on QOL in these types of pathologies, specifically on cardiorespiratory, neurological, and musculoskeletal pathologies (Li et al., 2014).

\section{HYDROTHERAPY}

The properties of water promote active movement, muscle relaxation, provide greater bodily and postural support, and stimulate strengthening by means of resistance of water, permitting the training of motor and sensory skills in subjects in water (Verhagen et al., 2012). This rehabilitation technique is based on the potential benefits of hydrodynamic principles such as bouyancy, resistance, relative density, viscosity, turbulence, and hydrostatic pressure and flow, in addition to providing sensory and physiological effects by means of the water temperature (Torres-Ronda and Del Alcázar, 2014).

Hydrotherapy (HT) is a technique that is applied as a complementary treatment in multiple pathologies. Evidence exists on the efficacy of this intervention with respect to pain diminution of pain and musculoskeletal function improvement in affectations such as low back pain, rheumatoid arthritis, OA, and fibromyalgia, and in neurological pathologies such as multiple sclerosis (Verhagen et al., 2012). In a meta-analysis published by Barker and colleagues that included 20 studies on the effectiveness of HT on musculoskeletal conditions, the authors found moderate effects on pain diminution and functionality (Barker et al., 2014).

\section{HYDROTHERAPY AND TC}

The technique and advantages of TC can theoretically be complemented with the benefits of HT. This concept has already been explored and although it is presently found in an experimentation phase, various studies have reported the efficacy of water-based postural exercise programs, suggesting positive effects on coordi- 
nation and equilibrium on combining these (Barker, 2014). When equilibrium training has been conducted on the floor, an individual's performance can be affected by lack of confidence, fear of falling, or joint pain. In an aquatic environment, the viscosity inherent in water serves as postural support, promoting confidence and reduction in fear of falling. The water temperature can be beneficial in pathologies that produce chronic pain, muscle contractions, or spasticity (Becker, 2009).

Novel techniques have been developed that combine the principles of TC and those of other disciplines similar to HT, or even techniques such as Ai Chi, aquatic exercise, which include TC, Qui Gong, Shiatsu or Watsu movements within an aquatic and musicotherapeutic environment. These programs have been tested in RCT as measures of the outcomes of pain, functionality, and disability, with significantly better results with respect to the group only engaging in breathing and relaxation exercises outside of the water (Castro-Sánchez et al., 2012).

Although Ai Chi is not synonymous with underwater TC, it does include movements of the latter with the potential benefits of HT, specifically in a pool, and variants of the technique have been developed that take advantage of the good aspects of both. Teixeira et al. (2011) conducted an RCT on the effects of Ai Chi on equilibrium and fear of falling in older adults. Thirty participants at the daycare center of a home for the fragile elderly were randomly assigned to an Ai Chi class or to a control group where they received customary caregiving. The intervention group received 16 sessions of Ai Chi during 6-week period. The results suggested that an $\mathrm{Ai}$ Chi program produces improvement in static and dynamic equilibrium in the elderly in comparison with conventional caregiving. The Ai Chi group maintained the same level of fear of falls, but in the control group, this increased (Teixeira et al., 2011).

In another RCT, with the objective to analyze the effects of an Ai Chi program on equilibrium in older adults, 54 subjects aged between 60 and $85 \mathrm{yr}$ were included who presented a high risk of falls. These subjects were distributed into two groups: the experimental group that participated in 12 sessions of $\mathrm{Ai}$ Chi, and the control group, the members of which carried out a conventional HT treatment in a pool. Equilibrium, joint mobility and pain were evaluated at the beginning and at the end of the intervention. Significant improvement in equilibrium in both groups was reported but, on performing the comparison between the groups, the experimental group was significantly better with respect to balance (Olabe-Sánchez and Martínez-Almagro, 2014).

Calandre et al. (2009) published an RCT in which the authors compared an Ai Chi technique with another involving floor exercises in persons with fibromyalgia; the authors concluded that there were no global differences between both groups, although there was significant improvement in symptoms and sleep quality in members of the Ai Chi group (Calandre et al., 2009). In a pilot study published by Pérez-de la Cruz and colleagues (2015), the authors analyzed the results of an Ai Chi program conducted in the water on QOL, depression, and pain in patients with fibromyalgia. Twenty patients were included, in whom functionality, pain, and QOL were measured; the intervention consisted of 20 sessions of the water-based program. The authors reported significant differences in the outcome variables in favor of the experimental group (Pérez-De la Cruz and Lambeck, 2015). Noh et al. (2008) reported another RCT in which they included 25 patients with chronic cerebrovascular-event sequelae who were assigned to two treatment groups: the experimental group underwent aquatic training, which included a combination of Ai Chi and Halliwick (another aquatic training technique) or a control group, with gymnastic floor exercises. The authors concluded that the aquatic therapy significantly improved equilibrium (Noh et al., 2008).

\section{CONCLUSIONS}

TC has demonstrated, in multiple studies, its usefulness on musculoskeletal system pathologies, due to its slow movements that involve the four limbs together with the regulation of breathing, postural control, and balance, and TC is now even recommended in some evidence-based clinical guidelines.

HT has been utilized with proven benefits derived from the mechanical properties of water that combine with the effects of the exercise, improving the muscle-skeletal conditions of the subjects.

Both treatment types involve the physical work of equilibrium, mobility, strength, coordination, and sensory input such as proprioception, with complementary advantages, which theoretically render them as possessing potential synergy in their benefits. In addition to this relaxation and breathing can provide a complete exercise, with low joint impact and strength work at the same time.

Combined TC and HT have been tested principally in older adults with risk of falls or fragility, and in some neurological or rheumatological pathologies. It is important to know whether TC practiced in water is useful in other chronic pathologies that cause pain and alterations in walking, such as knee or hip OA. For this, it is necessary to develop good-quality RCT in those included in conventional TC control groups, conventional HT groups, and in groups with both TC and HT combined, or conventional floor 
exercises (stretching, strengthening, equilibrium), in order to acquire general evidence that establishes whether there is an advantage on combining both techniques. In addition, it is necessary to carry out RCT with adequate samples sizes and greater follow-up to observe potential medium- and long-term benefits and to observe distinct techniques and modalities of TC as well as of HT.

\section{CONFLICT OF INTEREST}

No potential conflict of interest relevant to this article was reported.

\section{REFERENCES}

Barker AL, Talevski J, Morello RT, Brand CA, Rahmann AE, Urquhart DM. Effectiveness of aquatic exercise for musculoskeletal conditions: a meta-analysis. Arch Phys Med Rehabil 2014;95(9):1776-1786.

Becker BE. Aquatic therapy: scientific foundations and clinical rehabilitation applications. PM R 2009;1(9):859-872.

Calandre EP, Rodriguez-Claro ML, Rico-Villademoros F, Vilchez JS, Hidalgo J, Delgado-Rodriguez A. Effects of pool-based exercise in fibromyalgia symptomatology and sleep quality: a prospective randomized comparison between stretching and Ai Chi. Clin Exp Rheumatol 2009;27(5 Suppl 56):S21-S28.

Castro-Sánchez AM, Matarán-Peñarrocha GA, Lara-Palomo I, Saavedra-Hernández M, Arroyo-Morales M, Moreno-Lorenzo C. Hydrotherapy for the treatment of pain in people with multiplesclerosis: a randomized controlled trial. Evid Based Complement Alternat Med 2012;2012:473963.

Chen CH, Yen M, Fetzer S, Lo LH, Lam P. The effects of tai chi exercise on elders with osteoarthritis: a longitudinal study. Asian Nurs Res (Korean Soc Nurs Sci) 2008;2(4):235-241.

Escalante Y, Saavedra JM, Garcia-Hermoso A, Barbosa TM. Physical exercise and reduction of pain in adults with lower limb osteoarthritis: a systematic review. J Back Musculoskelet Rehabil 2010;23(4):175-186.

Fernandes L, Hagen KB, Bijlsma JW, Andreassen O, Christensen P, Conaghan PG, Doherty M, Geenen R, Hammond A, Kjeken I, Lohmander LS, Lund H, Mallen CD, Nava T, Oliver S, Pavelka K, Pitsillidou I, da Silva JA, de la Torre J, Zanoli G, VlietVlieland TP. EULAR recommendations for the non-pharmacological core management of hip and knee osteoarthritis. Ann Rheum Dis 2013;72(7):1125-1135.

Fong SM, Ng GY. The effects on sensorimotor performance and balance with Tai Chi training. Arch Phys Med Rehabil 2006;87(1):82-87.

Gatts SK, Woollacott MH. How Tai Chi improves balance: biomechanics of recovery to a walking slip in impaired seniors. Gait Posture 2007;
25(2):205-2014.

Gyllensten AL, Hui-Chan CW, Tsang WW. Stability limits, single-leg jump, and body awareness in older Tai Chi practitioners. Arch Phys Med Rehabil 2010;91(2):215-220.

Hall CD, Miszko T, Wolf SL. Effects of Tai Chi intervention on dual-task ability in older adults: a pilot study. Arch Phys Med Rehabil 2009; 90(3):525-529.

Hochberg MC, Altman RD, April KT, Benkhalti M, Guyatt G, McGowan J, Towheed T, Welch V, Wells G, Tugwell P. American College of Rheumatology 2012 recommendations for the use of non pharmacologic and pharmacologic therapies in osteoarthritis of the hand, hip, and knee. Arthritis Care Res (Hoboken) 2012;64(4):465-474.

Kang JW, Lee MS, Posadzki P, Ernst E. T'ai chi for the treatment of osteoarthritis: a systematic review and meta-analysis. BMJ Open 2011;1(1): e000035.

Lan C, Chen SY, Lai JS, Wong AM. Tai Chi Chuan in Medicine and Health Promotion. Evid Based Complement Alternat Med 2013;2013:502131.

Lan C, Chen SY, Lai JS, Wong MK. Heart rate responses and oxygen consumption during Tai Chi Chuan practice. Am J Chin Med 2001;29(3-4): 403-410.

Li G, Yuan H, Zhang W. Effects of Tai Chi on health related quality of life in patients with chronic conditions: a systematic review of randomized controlled trials. Complement Ther Med 2014;22(4):743-755.

Mat S, Tan MP, Kamaruzzaman SB, Ng CT. Physical therapies for improving balance and reducing falls risk in osteoarthritis of the knee: a systematic review. Age Ageing 2015;44(1):16-24.

McAlindon TE, Bannuru RR, Sullivan MC, Arden NK, Berenbaum F, Bierma-Zeinstra SM, Hawker GA, Henrotin Y, Hunter DJ, Kawaguchi H, Kwoh K, Lohmander S, Rannou F, Roos EM, Underwood M. OARSI guidelines for the non-surgical management of knee osteoarthritis. Osteoarthritis Cartilage 2014;22(3):363-88.

Noh DK, Lim JY, Shin HI, Paik NJ. The effect of aquatic therapy on postural balance and muscle strength in stroke survivors-a randomized controlled pilot trial. Clin Rehabil 2008;22(10-11):966-976.

Olabe-Sánchez PJ, Martínez-Almagro A. Repercusión del Ai Chi en el equilibrio de las personas mayores. Eur J Investig Health Psychol Educ 2014;4(3):247-256.

Pérez-De la Cruz S, Lambeck J. Effects of a programme of aquatic Ai Chi exercise in patients with fibromyalgia. A pilot study. Rev Neurol 2015;60:59-65.

Schneider D, Leung R. Metabolic and cardiorespiratory responses to the performance of Wing Chun and T'ai Chi Chuan exercise. Int J Sports Med 1991;12(3):319-323.

Teixeira R, PérezL, Lambeck J, Neto F. The influence of Ai Chi on balance and fear falling in older adults: a randomized clinical trial. Physiother- 
apy 2011:97 Supl 1:eS18-eS1415.

Torres-Ronda L, Del Alcázar XS. The properties of water and their applications for training. J Hum Kinet 2014;44:237-248.

Verhagen AP, Cardoso JR, Bierma-Zeinstra SM. Aquatic exercise \& balneotherapy in musculoskeletal conditions. Best Pract Res Clin Rheumatol 2012;26(3):335-343.

Wang C, Iversen MD, McAlindon T, Harvey WF, Wong JB, Fielding RA, Driban JB, Price LL, Rones R, Gamache T y Schmid CH. Assessing the comparative effectiveness of Tai Chi versus physical therapy for knee osteoarthritis: design and rationale for a randomized trial. BMC Complement Alter Med 2014;14:333.

Wang C. Role of Tai Chi in the treatment of rheumatologic diseases. Curr
Rheumatol Rep 2012;14(6):598-603.

Wang C. Tai Chi and rheumatic diseases. Rheum Dis Clin North Am 2011;37(1):19-32.

Ye J, Cai S, Zhong W, Cai S, Zheng Q. Effects of Tai Chi for patients with knee osteoarthritis: a systematic review. J Phys Ther Sci 2014;26(7):11331137.

Zhang L, Layne C, Lowder T, Liu J. A review focused on the psychological effectiveness of Tai Chi on different populations. Evid Based Complement Alternat Med 2012;2012:678107.

Zhuo D, Shephard RJ, Plyley MJ, Davis GM. Cardiorespiratory and metabolic responses during Tai Chi Chuan exercise. Can J Appl Sport Sci 1984;9(1):7-10 